\section{Movilidad, accidentalidad por tránsito y sus factores asociados en estudiantes universitarios de Guatemala}

\author{
Mobility, traffic accidents, and associated factors \\ among Guatemalan university students
}

\author{
Mobilidade, acidentes de trânsito e fatores \\ associados entre estudantes universitários \\ da Guatemala
}

\begin{abstract}
The aim of this study was to identify and quantify the association between the amount of driving (km/year), traffic accidents, and other factors among university students in Guatemala. A cross-sectional study was performed during the 2010-2011 school year in a sample of 1,016 drivers who completed a self-administered questionnaire that assessed mobility patterns, use of safety accessories, driving style, and automobile crashes. The results showed a positive association between amount of driving and greater involvement in risky driving (adjusted regression coefficient 3.25, 95\% CI: 2.23-4.27, for the highest level of exposure). More frequent involvement in risky driving and older age showed the strongest associations with traffic accidents. Although the amount of driving was positively associated with a higher accident rate, most of this association was found to be mediated by involvement in risky driving practices.
\end{abstract}

Traffic Accidents; Young Adults; Risk-Taking
Sandra Rodríguez-Guzmán 1

Eladio Jiménez-Mejías 2

Virginia Martínez-Ruiz 2

Fernando Lupiáñez-Tapia 2

Pablo Lardelli-Claret 2

José Juan Jiménez-Moleón 2

\section{Resumen}

El objetivo fue identificar y cuantificar la asociación entre la intensidad de exposición ( $\mathrm{km} / a \tilde{n} o$ recorridos), la accidentalidad y sus factores asociados en universitarios de Guatemala. Se realizó un estudio trasversal durante el curso 20102011, sobre una muestra de 1.016 conductores, quienes cumplimentaron un cuestionario autoadministrado que valoraba: patrones de movilidad, uso de dispositivos de seguridad, estilos de conducción y accidentalidad. Se obtuvieron asociaciones positivas entre la intensidad de exposición y la mayor implicación en circunstancias de riesgo al volante (coeficiente de regresión ajustado de 3,25, IC95\%: 2,23-4,27, para las mayores exposiciones). Tanto una mayor implicación en tales circunstancias, como una mayor edad, fueron las variables más fuertemente asociadas con la mayor accidentalidad. Pese a que la intensidad de exposición se asocia positivamente con una mayor accidentalidad, se constató que la mayor parte de dicha asociación está mediada por una mayor implicación en circunstancias de riesgo al volante.

Accidentes de Tránsito; Adulto Joven; Asunción de Riesgos 


\section{Introducción}

Hoy en día es sobradamente conocido que las lesiones por tránsito constituyen un problema de salud pública de primera índole. No en vano, representan la primera causa de mortalidad en todo el mundo entre los 15 a los 29 años, en especial entre los varones 1 . A ello han contribuido, entre otros factores, el exceso de velocidad, la conducción bajo los efectos del alcohol, la búsqueda de nuevas sensaciones o la escasa percepción de riesgo, tradicionalmente atribuidos a los jóvenes conductores 2,3,4.

Desde el ámbito de la epidemiología analítica, las lesiones por tránsito han dejado de percibirse como hechos fortuitos e impredecibles, para concebirse como el resultado final de una serie de acontecimientos secuencialmente ordenados en una cadena causal compuesta por cuatro eslabones: (1) movilidad o exposición, entendida en este trabajo como el número de kilómetrosaño recorridos, (2) accidentalidad, (3) lesividad y (4) desenlace (incapacidad residual o muerte) 5. Este planteamiento secuencial permite conocer el efecto de los diversos factores o marcadores de riesgo sobre cada uno de los mencionados eslabones que, finalmente, son los responsables de que un sujeto se accidente, resulte lesionado o, por desgracia, muera víctima de un accidente de tránsito.

En la actualidad, la mayor parte de la investigación epidemiológica sobre las lesiones por tránsito se centra en el estudio de los últimos eslabones de la mencionada cadena: la lesividad y la mortalidad 6,7. Esto es debido, entre otras razones, a la mayor disponibilidad de datos procedentes de fuentes secundarias (registros policiales, informes de urgencias y bases de datos de altas hospitalarias, entre otros). En Guatemala, uno de los países con mayores tasas de mortalidad por colisiones de tránsito de la Región de las Américas -14,7/100.000 habitantes según datos de la Organización Mundial de la Salud (OMS) de 2007 8-, existe una tendencia a su infra-notificación, así como una baja fiabilidad de la información al respecto, al no existir un registro unificado ${ }^{9}$. Además, los datos que se recogen están supeditados a que el evento haya sido lo suficientemente importante en términos de daño material y/o humano, como para haber requerido la intervención policial o, en su caso, de los servicios sanitarios. Todo ello hace que, probablemente, su número sea aún mayor según el Consejo de Prevención de Accidentes y Educación Vial Guatemalteco (CONPREVE) 10.

Por otra parte, los estudios basados en exclusiva en fuentes secundarias no permiten conocer los factores de riesgo asociados a los primeros eslabones de la mencionada cadena epidemiológica 11, lo que ha relegado a un segundo plano el estudio de los factores asociados a la exposición y a la accidentalidad y son escasos o, en el caso de Guatemala, inexistentes, los trabajos sobre la epidemiología analítica de las lesiones por tránsito que aborden de forma integral el estudio de dicha cadena causal 12,13. Esta limitación tiene, por desgracia, importantes consecuencias a la hora de cuantificar el verdadero papel de algunos factores o marcadores de riesgo de las lesiones por tránsito, por lo que se hace necesaria una primera aproximación a este fenómeno hasta ahora escasamente estudiado en Guatemala, partiendo de fuentes primarias y centrado en el estrato de edad con mayor impacto en términos de morbimortalidad.

Por todo ello, el presente estudio tiene por objetivo identificar y cuantificar la asociación entre la intensidad de exposición $(\mathrm{km} /$ año recorridos) y la accidentalidad, así como el posible papel mediador de los estilos de conducción de riesgo en la asociación anterior, en jóvenes estudiantes universitarios de Guatemala.

\section{Material y métodos}

Se diseñó un estudio transversal, tomando como población de estudio alumnos que cursaban las titulaciones de Médico y Cirujano, Técnico en Administración de Empresas, Licenciado en Ciencias Jurídicas y Sociales, Abogado y Notario, Auditor Técnico, Contador Público y Periodista Profesional, en el Centro Regional Universitario de Oriente (Sede Campus CURONI) de la Universidad Pública de San Carlos en Guatemala (USAC), durante el cuso académico 2010-2011. La elección de este Campus Universitario, así como las referidas titulaciones, se llevó a cabo por contar con uno de los mayores números de estudiantes inscritos en la USAC. Dicho número ascendió para el total de titulaciones impartidas en este campus, durante el curso académico 2010 2011, a 3.623 estudiantes 14 .

Algún día de las dos primeras semanas lectivas, en las que la asistencia a clase por parte del alumnado es mayor, los profesores correspondientes informaban a los estudiantes sobre el objetivo del estudio y repartían un cuestionario autoadministrado anónimo y de cumplimentación voluntaria y desinteresada. En total, el cuestionario fue cumplimentado por 1.271 alumnos, de los que 1.016 (el 79,9\%), manifestaron haber conducido un carro durante el año anterior a la encuesta. Los conductores de otros vehículos a motor como motocicletas, ciclomotores, etc. fueron igualmente incluidos, siempre y cuando 
hubiesen conducido además un carro durante el último año, pues fue este último el criterio que definió la muestra final de nuestro estudio.

El cuestionario empleado -Cuestionario sobre Movilidad, Accidentalidad por Tráfico y Circunstancias Asociadas (MATCA)-, consta de cinco bloques: I: Datos demográficos; II: Intensidad de exposición según tipo de usuario $(\mathrm{km}$. recorridos al año, estratificados originalmente en ocho categorías y, posteriormente, reagrupados en cuatro para los conductores de carro: < 500, 500-999, 1.000-4.999 y 5.000 o más km.); III: Frecuencia de uso de cinturón de seguridad estratificado por posición en el vehículo (conductor o pasajero) y tipo de vía (carretera o zona urbana); IV: Datos para conductores de carro: edad de obtención del permiso e implicación durante el mes anterior a la encuesta, en 25 diferentes circunstancias al volante de respuesta dicotómica (Sí/No) tales como: "Conducir de noche", "Sobrepasar la velocidad permitida”, "Conducir con sueño”, "No respetar un semáforo”, etc. (Tabla 1). A partir de esta última información, se construyó un índice de riesgo, sumando la implicación en aquellas circunstancias más claramente asociadas a un mayor riesgo de sufrir un accidente, según la literatura. En dicho índice, no fueron incluidas las circunstancias: "Conducir de noche", "Conducir solo" ni "Conducir con meteorología adversa" por no existir un consenso en la literatura sobre su carácter o no de circunstancias de riesgo en el mismo nivel que el resto de circunstancias incluidas 15,16,17. Finalmente, el bloque V contempló los accidentes de tráfico sufridos en el año anterior a la encuesta y, para el último accidente, diversas características del mismo

Regresión logística: frecuencia de implicación en cada circunstancia y asociación cruda y ajustada entre tal implicación y sufrir un accidente. Guatemala, 2010-2011.

\begin{tabular}{|c|c|c|c|c|c|}
\hline \multirow[t]{2}{*}{ Circunstancias } & \multicolumn{5}{|c|}{ Conductores accidentados } \\
\hline & $\%$ & ORc & IC95\% & $\mathrm{ORa}$ * & IC95\% \\
\hline Conducir de noche & 83,1 & 1,42 & $0,94-2,16$ & 1,23 & $0,76-1,97$ \\
\hline Sobrepasar la velocidad permitida & 52,6 & 1,92 & $1,43-2,60$ & 1,70 & $1,01-3,62$ \\
\hline Conducir con sueño & 52,9 & 1,57 & $1,17-2,12$ & 1,49 & $1,08-2,06$ \\
\hline No respetar el semáforo & 22,6 & 1,63 & $1,17-2,26$ & 1,49 & $1,05-2,14$ \\
\hline Conducir tras consumir alcohol & 31,7 & 1,50 & $1,11-2,03$ & 1,51 & $1,06-2,14$ \\
\hline Conducir solo & 91,5 & 5,53 & $2,21-13,80$ & 4,40 & $1,54-12,51$ \\
\hline Conducir tras consumir drogas & 2,3 & 2,09 & $0,89-4,88$ & 1,93 & $0,73-5,07$ \\
\hline Conducir con meteorología adversa & 87,8 & 2,48 & $1,41-4,34$ & 1,82 & $0,99-3,34$ \\
\hline Conducir usando el teléfono móvil & 74,4 & 2,60 & $1,75-3,88$ & 2,18 & $1,42-3,36$ \\
\hline No respetar el STOP & 17,5 & 1,37 & $0,96-1,98$ & 1,24 & $0,83-1,86$ \\
\hline Llevar a pasajeros sin cinturón & 45,6 & 1,60 & $1,20-2,15$ & 1,53 & $1,11-2,10$ \\
\hline Conducir sin cinturón & 23,9 & 1,28 & $0,92-1,78$ & 1,13 & $0,79-1,62$ \\
\hline Haber recibido una multa & 19,0 & 2,26 & $1,61-3,17$ & 2,23 & $1,54-3,23$ \\
\hline Conducir ebrio & 16,7 & 1,16 & $0,79-1,69$ & 1,25 & $0,81-1,92$ \\
\hline No respetar un paso peatonal & 15,5 & 1,17 & $0,78-1,72$ & 1,04 & $0,68-1,59$ \\
\hline Le han comentado que conduce deprisa & 30,7 & 1,49 & $1,10-2,01$ & 1,29 & $0,92-1,79$ \\
\hline Distraerse al volante & 47,1 & 2,22 & $1,65-3,00$ & 2,24 & $1,62-3,10$ \\
\hline Fumar conduciendo & 21,1 & 0,99 & $0,69-1,42$ & 1,01 & $0,68-1,51$ \\
\hline Escuchar la radio y cambiar dial & 82,5 & 1,91 & $1,23-2,96$ & 1,71 & $1,06-2,74$ \\
\hline Cambiar el CD conduciendo & 50,7 & 1,43 & $1,07-1,92$ & 1,47 & $1,06-2,03$ \\
\hline Comer conduciendo & 60,9 & 1,66 & $1,21-2,26$ & 1,35 & $0,96-1,88$ \\
\hline Conducir más de dos horas sin descanso & 38,3 & 1,46 & $1,09-1,96$ & 1,36 & $0,98-1,88$ \\
\hline Tocar el claxon injustificadamente & 24,3 & 1,55 & $1,12-2,13$ & 1,42 & $1,01-2,01$ \\
\hline Discutir con otros conductores & 25,0 & 1,41 & $1,02-2,00$ & 1,34 & $0,94-1,91$ \\
\hline Adelantar indebidamente & 25,3 & 1,91 & $1,40-2,62$ & 1,82 & $1,29-2,58$ \\
\hline
\end{tabular}

IC95\%: intervalo del 95\% de confianza; ORa: odds ratio ajustada; ORc: odds ratio cruda.

* OR ajustadas por sexo, edad, antigüedad en el permiso e intensidad de exposición (km/año). 
(tipo de usuario, severidad, responsabilidad). Con objeto de responder a los objetivos planteados, únicamente fueron considerados los accidentes en los que el encuestado viajaba como conductor del carro accidentado. Dado el escaso número de encuestados que manifestaron haber sufrido lesiones a consecuencia del accidente, $(<1 \%)$ no ha sido posible analizar la lesividad.

De los cinco bloques anteriores, la fiabilidad del segundo fue previamente medida en una muestra de 90 alumnos y médicos residentes de primer año del Hospital Clínico Universitario de Granada (España), contrastándola con la obtenida a través de una versión modificada del Driving Habits Questionnaire, desarrollado por Owsley et al. 18, obteniéndose una concordancia aceptable entre ambos instrumentos. Con respecto al bloque IV, la validez de convergencia y de criterio de las circunstancias planteadas (seleccionadas por un panel de expertos a partir de una revisión exhaustiva de la bibliografía) 19 , ha sido validada previamente a partir de la muestra de estudiantes universitarios de Granada 20. Para poder emplear dicho cuestionario en nuestra población, realizamos ligeras modificaciones en la formulación de alguno de sus ítems con el objeto de adaptarlos a las características de Guatemala.

Para el análisis de las asociaciones entre variables, aunque nuestro estudio es de tipo trans- versal y no permite verificar hipótesis causales, nos pareció oportuno partir de un planteamiento causal. Para ello construimos un Gráfico Acíclico Dirigido (DAG) 21 (Figura 1), basándonos en la citada cadena causal de las lesiones por tránsito. En dicho DAG pueden diferenciarse cuatro grupos de variables: la intensidad de exposición, la accidentalidad, un grupo de posibles mediadores entre las dos variables anteriores, constituido por la implicación en distintas circunstancias durante la conducción y el no uso del cinturón de seguridad (esta última variable, a tenor de la literatura 22 , puede considerarse un marcador de riesgo de otras circunstancias o estilos de conducción asociados a una mayor accidentalidad) $\mathrm{y}$, finalmente, un conjunto de posibles confusores de las asociaciones anteriores, en el que incluimos la edad, el sexo y la antigüedad del permiso de conducir.

Partiendo de este planteamiento analizamos, en primer lugar, el efecto que las variables sexo, edad, antigüedad del permiso de conducir carro e intensidad de exposición, tuvieron sobre la implicación en circunstancias de riesgo durante el último mes. Posteriormente, se valoró la frecuencia de implicación por parte de los conductores en cada una de las circunstancias estudiadas, así como la asociación cruda y ajustada entre tal implicación y la accidentalidad durante el último

Figura 1

Gráfico acíclico dirigido de las lesiones por tráfico.

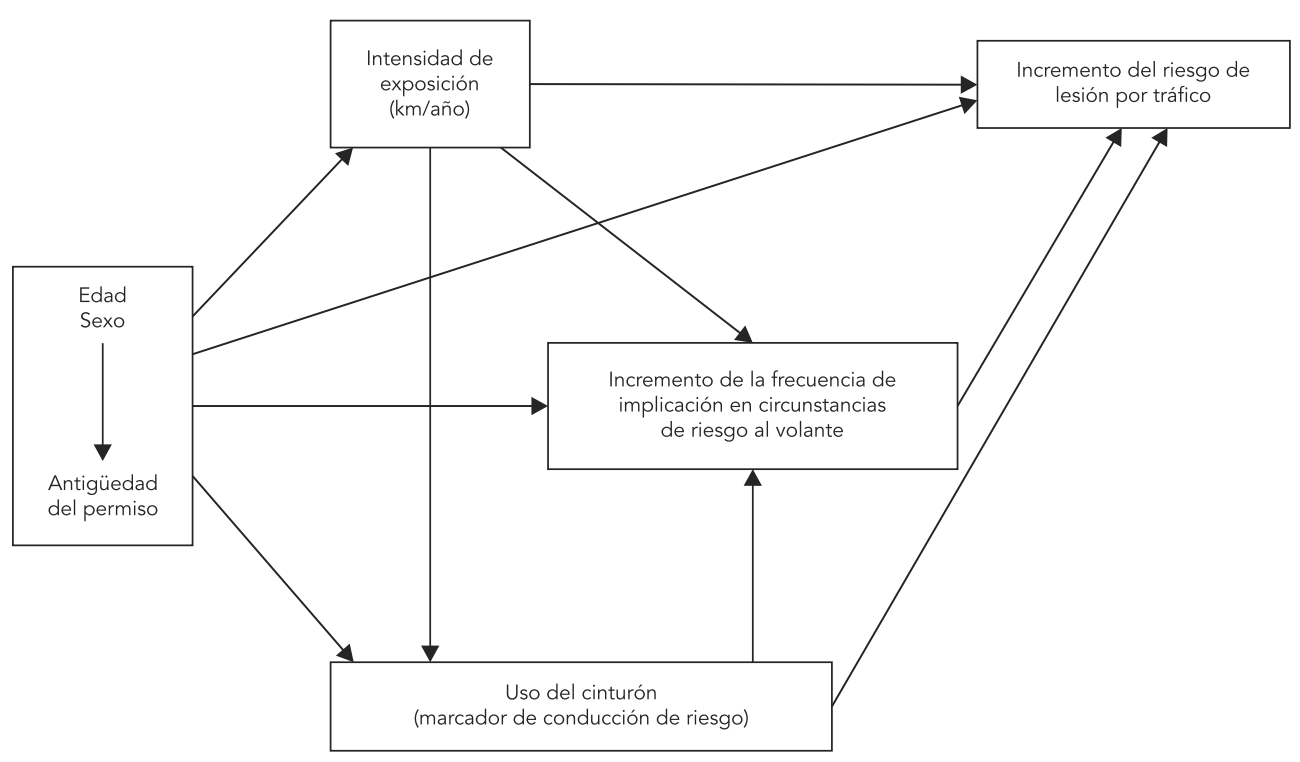


año. Finalmente, se valoró el efecto que sobre la accidentalidad tenían las citadas variables, además del no uso del cinturón y el número de circunstancias de riesgo en las que el conductor se implicó durante el último mes.

Para estimar las asociaciones planteadas se calcularon odds ratio (OR) crudas y ajustadas mediante modelos de regresión logística (para variables dependientes dicotómicas, como la implicación o no en las diferentes circunstancias de conducción de riesgo o la accidentalidad) y de regresión lineal múltiple (para variables cuantitativas continuas como la puntuación en el índice de implicación en circunstancias de riesgo). La bondad de ajuste del modelo multivariante final fue evaluada mediante el test de Hosmer Lemeshow, que arrojó un valor de $\chi^{2}$ de 0,8655. El software empleado fue el paquete estadístico Stata, versión 11.0 (Stata Corp., College Station, Estados Unidos).

Para la realización del presente estudio se contó con la aprobación del Comité de Bioética de los centros implicados y se respetaron los principios éticos para investigaciones contemplados en la Declaración de Helsinki (2008).

\section{Resultados}

La Tabla 2 muestra la distribución de los conductores en función de las principales variables estudiadas. El 53,1\% de ellos fueron mujeres. La edad media fue de 20,7 años, con mediana de 20 y desviación típica de 2,3. El promedio de años en posesión del permiso de conducir carro fue de 3,3. La frecuencia de uso del cinturón de seguridad fue ligeramente superior en carretera, aunque no de forma estadísticamente significativa ( $p=0,863$ ). Finalmente, el $23,7 \%$ de los conductores encuestados manifestaron haber sufrido al menos un accidente de tránsito en el último año.

En la Tabla 3 se muestra el efecto ajustado de la intensidad de exposición (km/año recorridos), del sexo, la edad y la antigüedad del permiso de conducir, sobre la frecuencia de implicación en el último mes en circunstancias de riesgo al volante. Puede observarse como, a mayor intensidad de exposición y mayor antigüedad del permiso, mayor fue la frecuencia de implicación en tales circunstancias, con gradientes dosis-respuesta positivos para ambas variables. Las mujeres, por su parte, se implicaron sensiblemente menos que los varones en tales circunstancias (coeficiente de regresión: -1,69; IC95\%: [-2,26]-[-1,12]).

La Tabla 1 muestra la frecuencia de implicación de los conductores durante el último mes en cada una de las circunstancias exploradas, así como la asociación cruda y ajustada por sexo, edad
Tabla 2

Distribución del sexo, edad, antigüedad del permiso de automóvil, uso del cinturón y accidentalidad entre los conductores. Guatemala, 2010-2011.

\begin{tabular}{|c|c|c|}
\hline Variable/Categorías & $\mathbf{n}$ & $\%$ * \\
\hline \multicolumn{3}{|l|}{ Sexo } \\
\hline Varón & 474 & 46,9 \\
\hline Mujer & 537 & 53,1 \\
\hline Total & 1.011 & 100,0 \\
\hline \multicolumn{3}{|l|}{ Edad (años) } \\
\hline$\leq 20$ & 274 & 28,3 \\
\hline $21-22$ & 433 & 44,7 \\
\hline $23-24$ & 189 & 19,5 \\
\hline$\geq 25$ & 72 & 7,4 \\
\hline Total & 968 & 100,0 \\
\hline \multicolumn{3}{|c|}{ Antigüedad del permiso de coche (años) } \\
\hline$\leq 1$ & 173 & 19,4 \\
\hline $2-3$ & 373 & 41,7 \\
\hline $4-5$ & 219 & 24,5 \\
\hline$\geq 6$ & 129 & 14,4 \\
\hline Total & 894 & 100,0 \\
\hline \multicolumn{3}{|c|}{ Uso del cinturón en carretera } \\
\hline Siempre & 756 & 77,7 \\
\hline No siempre & 217 & 22,3 \\
\hline Total & 973 & 100,0 \\
\hline \multicolumn{3}{|c|}{ Uso del cinturón en ciudad } \\
\hline Siempre & 750 & 74,3 \\
\hline No siempre & 260 & 25,7 \\
\hline Total & 1.010 & 100,0 \\
\hline \multicolumn{3}{|c|}{ Algún accidente en el último año } \\
\hline Sí & 241 & 23,7 \\
\hline No & 775 & 76,3 \\
\hline Total & 1.016 & 100,0 \\
\hline
\end{tabular}

* Porcentajes por columnas, sobre el total de valores no faltantes para cada variable.

y antigüedad del permiso, entre tal implicación y haber sufrido algún accidente en el último año. Puede observarse como las mayores implicaciones se dieron en las circunstancias: "conducir solo", "conducir de noche", "conducir bajo meteorología adversa" o "conducir usando el teléfono móvil”. Todas ellas con frecuencias superiores al 70\%. Otras, como "comer mientras se conduce", "conducir con sueño", "conducir por encima de la velocidad autorizada", "distraerse al volante" o “llevar a los pasajeros sin cinturón”, presentaron frecuencias de implicación entre 45 y $53 \%$.

A excepción de "fumar mientras se conduce", el resto de circunstancias exploradas se asociaron positivamente con una mayor accidentalidad. 
Regresión lineal múltiple: asociación ajustada entre exposición, sexo, edad y antigüedad del permiso e implicarse en circunstancias de riesgo. Guatemala, 2010-2011.

\begin{tabular}{|c|c|c|}
\hline \multirow[t]{2}{*}{ Variables/Categorías } & \multicolumn{2}{|c|}{ Implicación en circunstancias de riesgo * } \\
\hline & Coeficientes ajustados & IC95\% \\
\hline \multicolumn{3}{|l|}{ Exposición (km/año) ** } \\
\hline $500-999$ & 1,73 & $0,78-2,70$ \\
\hline $1.000-4.999$ & 2,42 & $1,39-3,44$ \\
\hline$\geq 5.000$ & 3,25 & $2,23-4,27$ \\
\hline \multicolumn{3}{|l|}{ Sexo $* \star \star$} \\
\hline Mujer & $-1,69$ & $(-2,26)-(-1,12)$ \\
\hline \multicolumn{3}{|l|}{ Edad (años) \# } \\
\hline $21-22$ & $-0,16$ & $-0,91-0,59$ \\
\hline $23-24$ & $-1,05$ & $-2,18-0,74$ \\
\hline$\geq 25$ & $-1,25$ & $-2,82-0,32$ \\
\hline \multicolumn{3}{|c|}{ Antigüedad del permiso (años) \#\# } \\
\hline $2-3$ & 1,02 & $0,21-1,83$ \\
\hline $4-5$ & 2,28 & $1,23-3,32$ \\
\hline$\geq 6$ & 3,14 & $1,66-4,61$ \\
\hline
\end{tabular}

IC95\%: intervalo del 95\% de confianza.

Categorías de referencia de la regresión lineal (variable dependiente):

* Implicarse en circunstancias de riesgo al volante.

Categorías de referencia de la regresión lineal (variables independientes):

** Exposición $<500 \mathrm{~km} /$ año;

$\star \star \star$ Sexo masculino;

\# Edad $\leq 20$ años;

\#\# Antigüedad del permiso $\leq 1$ año.

Aún tras ajustar por sexo, edad, antigüedad del permiso e intensidad de exposición, la mayor parte de las circunstancias exploradas, mantuvieron su asociación con una mayor accidentalidad, en especial: "conducir solo", "usar el móvil", "distraerse al volante", "haber recibido una multa por parte de la policía”, "adelantar indebidamente", "conducir por encima de la velocidad autorizada" y "conducir tras haber consumido alcohol". Todas ellas con ORa superiores a 1,50.

Finalmente, la Tabla 4 muestra un modelo de regresión logística que analiza el efecto sobre la accidentalidad de la intensidad de exposición, el sexo, la edad, la antigüedad del permiso, el no uso del cinturón y el número de circunstancias de riesgo en las que los conductores se implicaron durante el último mes. Las estimaciones ajustadas muestran que las variables que mantuvieron una asociación positiva con la accidentalidad fueron: una edad mayor o igual a 25 años y la implicación en circunstancias de riesgo al volante, con ORa de 3,84, IC95\%: 1,47-9,87 y de 1,11, IC95\%: 1,05-1,17, respectivamente. Por su parte, los conductores con una antigüedad del permiso mayor o igual a los 6 años, presentaron una menor accidentalidad con respecto a los más nóveles.

\section{Discusión}

La muestra se compuso mayoritariamente de sujetos jóvenes. El 90\% de los conductores tenían, en el momento de la encuesta, una edad menor o igual a 23 años. Ello explica los pocos años de antigüedad en posesión del permiso de conducir (3 años de media) y el predominio de las bajas intensidades de exposición; más del 70\% de los conductores, había recorrido menos de $5.000 \mathrm{~km}$ durante el año anterior. Tales hallazgos son coincidentes con los observados por otros autores en poblaciones de jóvenes conductores 23,24 . La distribución por sexos está en consonancia con la documentada por el Instituto Universitario de la Mujer de la USAC 25. Resulta llamativo, no obstante que, en una población con una baja experiencia al volante y con un elevado nivel formativo, más del $22 \%$ refieran no usar siempre 
Regresión logística: asociación cruda y ajustada entre exposición, sexo, edad, antigüedad del permiso, uso del cinturón y número de circunstancias con la accidentalidad. Guatemala, 2010-2011.

\begin{tabular}{|c|c|c|c|c|}
\hline \multirow[t]{2}{*}{ Variables/Categorías } & \multicolumn{4}{|c|}{ Conductores accidentados * } \\
\hline & ORc & IC95\% & ORa & IC95\% \\
\hline \multicolumn{5}{|l|}{ Exposición (km/año) ** } \\
\hline $500-999$ & 3,40 & $1,90-6,09$ & 1,78 & $0,89-3,55$ \\
\hline $1.000-4.999$ & 2,99 & $1,63-5,48$ & 1,44 & $0,70-2,99$ \\
\hline$\geq 5.000$ & 2,66 & $1,46-4,85$ & 1,27 & $0,61-2,98$ \\
\hline \multicolumn{5}{|l|}{ Sexo *** } \\
\hline Mujer & 0,74 & $0,56-0,99$ & 0,88 & $0,62-2,63$ \\
\hline \multicolumn{5}{|l|}{ Edad (años) \# } \\
\hline $21-22$ & 1,21 & $0,85-1,74$ & 1,07 & $0,67-1,70$ \\
\hline $23-24$ & 0,96 & $0,61-1,50$ & 1,85 & $0,94-3,62$ \\
\hline$\geq 25$ & 1,37 & $0,76-2,47$ & 3,81 & $1,47-9,87$ \\
\hline \multicolumn{5}{|c|}{ Antigüedad del permiso (años) \#\# } \\
\hline $2-3$ & 1,85 & $1,20-2,85$ & 1,39 & $0,82-2,36$ \\
\hline $4-5$ & 1,12 & $0,68-1,83$ & 0,58 & $0,29-1,15$ \\
\hline$\geq 6$ & 0,98 & $0,55-1,75$ & 0,27 & $0,11-0,70$ \\
\hline \multicolumn{5}{|c|}{ Uso del cinturón en carretera \#\#\# } \\
\hline No siempre & 1,02 & $0,71-1,45$ & 0,79 & $0,45-1,38$ \\
\hline \multicolumn{5}{|c|}{ Uso del cinturón en ciudad \#\#\# } \\
\hline No siempre & 1,12 & $0,81-1,55$ & 0,99 & $0,57-1,69$ \\
\hline \multicolumn{5}{|c|}{ Número de circunstancias de riesgo $\S$} \\
\hline Cada circunstancia & 1,11 & $1,07-1,14$ & 1,11 & $1,07-1,17$ \\
\hline
\end{tabular}

IC95\%: intervalo del 95\% de confianza; ORa: odds ratio ajustada; ORc: odds ratio cruda.

Categorías de referencia de la regresión lineal (variable dependiente):

* Haber sufrido algún accidente.

Categorías de referencia de la regresión lineal (variables independientes):

** Exposición < 500km/año;

*** Sexo masculino;

\# Edad $\leq 20$ años;

\#\# Antigüedad del permiso $\leq 1$ año;

\#\#\# Uso siempre del cinturón en carretera o ciudad;

$\S$ No implicarse en cada circunstancia.

el cinturón de seguridad, en especial en ciudad, pese a ser de uso obligatorio. Desgraciadamente, no se trata de una conducta infrecuente entre los jóvenes, y son varios los estudios realizados en diversos países que cifran el no uso de cinturón por parte de éstos entre un 20 y un $40 \%$ 26,27,28.

En torno al $24 \%$ de los conductores encuestados manifestó haber sufrido un accidente durante el año anterior. Esta cifra es ligeramente superior a la reportada en población joven de Guatemala en un estudio descriptivo realizado por Escobedo-Qujivix 9. No obstante, hemos de ser muy cautos al comparar ambas cifras, pues dicho estudio se realizó en población general y los rangos de edad definidos no coinciden exactamente con los empleados en el nuestro.
En relación con el hecho de que las mayores intensidades de exposición se asocien positivamente con la frecuencia de accidentes, son múltiples los trabajos que, al igual que el nuestro, han detectado esta asociación en jóvenes conductores 29,39,31. Se trata de un hallazgo esperable ligado al hecho de que, como cualquier otro fenómeno incidente, su frecuencia de aparición depende de la duración del tiempo a riesgo. Dicha asociación, sin embargo, se atenúa considerablemente cuando se ajusta en función del sexo, la edad, la antigüedad y las que hemos llamado variables intermediarias: el no uso del cinturón y la implicación en circunstancias de riesgo (Tabla 4). Desde una perspectiva causal, ello querría decir que buena parte de la asociación entre 
exposición y accidentalidad estaría mediada por este conjunto de variables. Así, deberíamos esperar una asociación consistente tanto entre la exposición y la implicación en dichas variables, como entre estas últimas y el accidente. Sin embargo, tampoco podemos descartar en dichas asociaciones el posible efecto de factores no medidos y la consiguiente confusión residual que ello introduciría, lo que nos obliga a interpretar estos resultados también con cautela.

Con respecto a la primera de estas asociaciones, nuestros datos revelan una relación dosisrespuesta directa entre la intensidad de exposición y la frecuencia de implicación en circunstancias de conducción de riesgo, en especial entre los conductores con elevadas intensidades de exposición. Usar el móvil mientras se conduce, conducir con sueño o cansancio, conducir por encima de la velocidad autorizada y distraerse al volante fueron las circunstancias con mayores frecuencias de implicación entre nuestros conductores. Este hecho ya ha sido documentado por la literatura en conductores jóvenes de otros países 3,32,33. Así, un reciente estudio realizado en jóvenes conductores australianos detectó que, entre los principales comportamientos de riesgo al volante se encontraban: el exceso de velocidad, el uso del móvil, conducir cansado, llevar pasajeros sin cinturón, conducir solo, conducir de noche, bajo meteorología adversa y conducir bajo los efectos del alcohol u otras drogas 16 .

Respecto a la segunda de las asociaciones planteada: la implicación en las diferentes circunstancias de conducción de riesgo y la accidentalidad, obtuvimos una asociación consistente para la mayoría de ellas, en especial para: "distraerse al volante", "haber recibido una multa por parte de la policía”, "usar el móvil mientras se conduce", "adelantar de forma indebida” y "conducir por encima de la velocidad autorizada”. Todas estas circunstancias han sido descritas como factores de riesgo de accidente en diversos trabajos 34,35,36,37. En esta misma línea, Nabi et al. 38 demostraron en un estudio basado en la cohorte GAZEL que, tras ajustar por sexo y exposición, los principales factores de riesgo de sufrir un accidente fueron el exceso de velocidad, el uso de teléfono móvil al volante, las violaciones de las normas de seguridad vial y conducir con sueño. Otro estudio, realizado en universitarios de entre 18 a 23 años, demostró la existencia de un clúster formado por conductores con comportamientos de riesgo, en los que aparecían asociadas la elevada intensidad de exposición, una alta velocidad al volante, un mayor número de infracciones de las normas, haber recibido al menos una multa por parte de la policía y una mayor accidentalidad 31 .
Pese a que resulta difícil separar el efecto de la edad del de la antigüedad en el permiso, pues ambas variables se encuentran intrínsecamente asociadas 39, el análisis ajustado revela un comportamiento parcialmente independiente y así, una mayor edad se asocia a una mayor accidentalidad con un gradiente dosis-respuesta, mientras que con la antigüedad, se observa el fenómeno opuesto. Probablemente, este hecho esté condicionado a la mayor percepción de riesgo al volante que aporta la experiencia, tal y como apuntan algunos autores 40 .

Somos conscientes de que nuestro estudio presenta diversas limitaciones que es necesario tener en cuenta a la hora de interpretar adecuadamente sus resultados. En primer lugar su carácter transversal, que impide relacionar causalmente la accidentalidad con la exposición y con los patrones de conducción. Por otra parte, las características peculiares de la población de estudio (jóvenes universitarios conductores de carro y con escasa experiencia de conducción), que limitan la capacidad de extrapolar los patrones identificados a otras poblaciones de conductores jóvenes y, por supuesto, a conductores de otras edades.

Aunque la tasa de respuesta entre los alumnos fue muy elevada (siempre superior al $90 \%$ en todas las aulas), es cierto que no se incluyeron a los alumnos que no asistieron a clase al inicio del curso, lo que puede haber introducido un sesgo de selección de sentido incierto. Analizadas las diferencias por edad y sexo entre los participantes en el estudio con respecto a los que no lo hicieron, éstas no fueron relevantes. Además, los modelos multivariantes empleados han permitido al menos ajustar las asociaciones por los grandes determinantes de la accidentalidad por tráfico, dependientes de factores individuales como: edad, sexo, experiencia, intensidad de exposición y patrones de conducción de riesgo.

Por otro lado, el cuestionario MATCA ha sido validado en la población de estudiantes universitarios españoles, pero no así en Guatemala. En este sentido, la adaptación transcultural del cuestionario a una población teóricamente muy distinta de aquella para la que fue originalmente diseñado es más que cuestionable, aspecto éste que debe ser muy tenido en cuenta en futuros estudios. A ello se une el más que probable sesgo de información inherente a cualquier estudio basado en cuestionarios autoadministrados 41 . Sin embargo, pensamos que el carácter anónimo del cuestionario y la concordancia de nuestros resultados con los observados en la mayoría de estudios precedentes, es un argumento a favor de la validez de nuestros datos. 
Pese a las limitaciones descritas, nuestros resultados revelan que una mayor exposición al volante se asocia a una mayor frecuencia de accidentes de tránsito. Sin embargo, la mayor parte de esta asociación parece estar mediada por la implicación en conductas de riesgo, entre las que sobresalen las distracciones, la comisión de infracciones, el uso de teléfono móvil y el exceso de velocidad. Su estudio en muestras más heterogéneas podría esclarecer cuáles son las dianas más importantes sobre las que dirigir la prevención de este importante problema de salud pública de manera más eficiente.

\section{Resumo}

O objetivo foi identificar e quantificar a associação entre a intensidade de exposição (km/anos percorridos), a acidentalidade e os seus fatores em universitários da Guatemala. Realizou-se um estudo transversal durante o curso 2010-2011 sobre uma amostra de 1.016 condutores de veículos, que responderam a um questionário autoadministrado que valorizava: padrões de mobilidade, uso de dispositivos de segurança, maneira de condução e acidentalidade. Obtiveram-se associações positivas entre intensidade de exposição e maior implicância em circunstâncias de risco ao conduzir (coeficiente de regressão ajustado a 3,25; IC95\%: 2,23-4,27, para as maiores exposições). Tanto a maior implicância em ditas circunstâncias como uma maioridade foram as variáveis mais fortemente associadas com a maior acidentalidade. Apesar de a intensidade de exposição ser associada positivamente com uma maior acidentalidade, estabelecemos que a maior parte da dita associação está mediada pela maior implicância em circunstâncias de risco ao conduzir.

Acidentes de Trânsito; Adulto Jovem; Assunção de Riscos

\section{Colaboradores}

S. Rodríguez-Guzmán contribuyó con la recogida de datos y redacción del manuscrito. E. Jiménez-Mejías contribuyó con el diseño del estudio, redacción del manuscrito y revisión final. V. Martínez-Ruiz y F. Lupiáñez-Tapia contribuyeron con la búsqueda bibliográfica, análisis de datos y revisión final. P. Lardelli-Claret contribuyó con la concepción y diseño del estudio y revisión final. J. J. Jiménez-Moleón contribuyó con la revisión del manuscrito, supervisión del análisis de datos y lectura crítica.

\section{Agradecimientos}

A todos los estudiantes y profesores de la Universidad San Carlos de Guatemala que de manera desinteresada han participado en el presente estudio. 


\section{Referencias}

1. Organización Mundial de la Salud. Informe sobre la situación mundial de la seguridad vial: es hora de pasar a la acción. http://www.who.int/violen ce_injury_prevention/road_safety_status/report/ cover_and_front_matter_es.pdf (accedido el 29/ May/2013).

2. Giacomo C, Toledo T, Lotan T, Taubman O, Ari B. Modeling the behavior of novice young drivers during the first year after licensure. Accid Anal Prev 2010; 42:480-6.

3. Ivers R, Senserrick T, Boufous S, Stevenson M, Chen HY, Woodward M, et al. Novice drivers's risky behaviour, risk perception, and crash risk: finding from the DRIVE Study. Am J Public Health 2009; 99:1638-44.

4. Scott-Parker B, Watson B, King MJ, Hyde MK. A further exploration of sensation seeking propensity, reward sensitivity, depression, anxiety, and the risky behaviour of young novice drivers in a structural equation model. Accid Anal Prev 2013; 50:465-71.

5. Jiménez-Moleón JJ, Lardelli-Claret P. ¿Cómo puede ayudar la medicina? Epidemia de los accidentes de tráfico. Med Clin (Barc) 2007; 128:178-80.

6. Petridou ET, Yannis G, Terzidis A, Dessypris N, Germeni E, Evgenikos P, et al. Linking emergency medical department and road traffic police casualty data: a tool in assessing the burden of injuries in less resourced countries. Traffic Inj Prev 2009; 10:37-43.

7. Rios PAA, Mota ELA. Traffic deaths: recent evolution and regional differences in Bahia State, Brazil. Cad Saúde Pública 2013; 29:131-44.

8. Organización Mundial de la Salud. Informe sobre el estado de la seguridad vial en la región de las Américas. http://www.who.int/violence_injury_ prevention/road_safety_status/2009/gsrrs_paho. pdf (accedido el 02/Jun/2013).

9. Escobedo-Qujivix JM. Perfil epidemiológico de los accidentes de tránsito urbano [Tesis de Doctorado]. Guatemala: Universidad de San Carlos de Guatemala; 2011.

10. Ministerio de Comunicaciones, Infraestructura y Vivienda, Gobierno de la República de Guatemala. Operativo anual POA 2013. http://www.caminos. gob.gt/accesoalainformacion/docs/201302/Art 10Num05.pdf (accedido el 22/May/2013).

11. Jeffrey S, Stone DH, Blamey A, Clark D, Cooper C, Dickson K, et al. An evaluation of police reporting of road casualties. Inj Prev 2009; 15:13-8.

12. Montenegro-Alvizurez GA. Causas de la inexistencia de una política preventiva del estado, en relación a los hechos de tránsito, originados por el consumo de alcohol y estupefacientes [Tesis de Doctorado]. Guatemala: Universidad de San Carlos de Guatemala; 2008.

13. Papadakati M, Kontagiannis T, Tzamalouka G, Darviri C, Chliaoutakis J. Exploring the effects of lifestyle, sleep factors and driving behaviours on sleep-related road risk: a study of Greek drivers. Accid Anal Prev 2008; 40:2029-36.
14. Universidad de San Carlos de Guatemala. Estadísticas. Centros universitarios por sexo, 2010. http:// www.usac.edu.gt/estadisticas.php (accedido el 12/Jun/2013).

15. Lansdown TC. Individual differences and propensity to engage with in-vehicle distractions: a self report survey. Transp Res Part F Traffic Psychol Behav 2012; 15:1-8.

16. Scott-Parker B, Watson B, King MJ, Hyde MK. Confirmatory factor analysis of the behavior of young novice drivers scale (BYNDS). Accid Anal Prev 2012; 49:385-91.

17. Neyens DM, Boyle LN. Crash risk factors related to individuals sustaining and drivers following traumatic brain injuries. Accid Anal Prev 2012; 49: 266-73.

18. Owsley C, Stalvey B, Wells J, Sloane ME. Older drivers and cataract: driving habits and crash risk. J Gerontol A Biol Sci Med Sci 1999; 54:203-11.

19. Jiménez-Mejías E, Lardelli-Claret P, Amezcua-Prieto C, Jiménez-Moleón JJ. Cuestionarios sobre factores de riesgo de la exposición y la accidentalidad por tráfico. Una revisión. An Sist Sanit Navar 2011; 34:443-52.

20. Jiménez-Mejías E, Luna-del Castillo JD, AmezcuaPrieto C, Olvera-Porcel MC, Lardelli-Claret P, Jiménez-Moleón JJ. Diseño y validación de un cuestionario sobre patrones de conducción de riesgo en jóvenes. Rev Esp Salud Pública 2012; 86:71-84.

21. Hernán MA, Robins JM. Causal inference. http:// www.hsph.harvard.edu/miguel-hernan/causalinference-book/ (accedido el 19/Abr/2013).

22. Şimşekoğlu Ö, Lajunen T. Relationship of seat belt use to health and driver behaviors. Transp Res F Traffic Psychol Behav 2009; 12:235-41.

23. Vallaso S, Smart D, Sanson A, Harrison W, Harris A, Cockfield S, et al. Risky driving among young drivers: trends, precursors and correlates. Accid Anal Prev 2007; 39:444-58.

24. Cestac J, Paran F, Delhomme P. Young drivers' sensation seeking, subjective norms, and perceived and perceived behavioral control and their roles in predicting intention: How risk-taking motivations evolve gender and driving experience. Saf Sci 2011; 49:424-32.

25. Maldonado-Batres MI, Villagrán-Barahona MI, Borrayo-Morales AP. Acciones con enfoque de género que promueve la Universidad de San Carlos de Guatemala 2000-2004. http://digi.usac.edu.gt/ iumusac/sistemas/docs/accionesgenero.pdf (accedido el 12/Mar/2013).

26. Briggs NC, Lambert EW, Goldzweig IA, Levine RS, Warren RC. Driver and passenger seatbelt use among U.S. high school students. Am J Prev Med 2008; 35:224-49.

27. Sangowawa AO, Alagh BT, Ekanem SE, Ebong IP, Faseru B, Adekunle BJ, et al. An observational study of seatbelt use among vehicle occupants in Nigeria. Inj Prev 2010;16:85-9. 
28. Sarma KM, Carey RN, Kervick AA, Bimpeh Y. Psychological factors associated with indices of risky, reckless and cautious driving in a national sample of drivers in the Republic of Ireland. Accid Anal Prev 2013; 50:1226-35.

29. Laapotti S, Keskinen E, Hatakka K, Hernetkoski K, Katila A, Peräaho M, et al. Driving circumstances and accidents among novice drivers. Traffic Inj Prev 2006;7:232-7.

30. Lucidi F, Giannini AM, Sgalla R, Mallia L, Devoto A, Reichmann S. Young novice driver subtypes: relationship to driving violations, errors and lapses. Accid Anal Prev 2010; 42:1689-96.

31. Scott-Parker B, Watson B, King MJ, Hyde MK. Mileage, car ownership, experience of punishment avoidance, and the risky driving of young drivers. Traffic Inj Prev 2011;12:559-67.

32. Machin MA, Sankey KS. Relationships between young drivers' personality characteristics, risk perceptions, and driving behaviour. Accid Anal Prev 2008; 40:541-7.

33. Centers for Disease Control and Prevention. Mobile device use while driving: United States and seven European countries, 2011. MMWR Morb Mortal Wkly Rep 2013; 62:177-82.

34. Boufous S, Ivers R, Senserrick T, Stevenson M, Norton R, Williason A. Accuracy of self-report of on road crashes and traffic offences in a cohort of young drivers: the DRIVE study. Inj Prev 2010; 16:275-7.
35. Awadzi KD, Classen S, Hall A, Duncan RP, Garvan CW. Predictors of injury among younger and older adults in fatal motor vehicle crashes. Accid Anal Prev 2008; 40:1804-10.

36. Hassan HM, Abdel-Aty MA. Exploring the safety implications of young drivers' behavior, attitudes and perceptions. Accid Anal Prev 2013; 50:361-70.

37. Scott-Parker B, Hyde MK, Watson B, King MJ. Speeding by young novice drivers: What can personal characteristics and psychosocial theory add to our understanding? Accid Anal Prev 2013; 50:242-50.

38. Nabi H, Salmi LR, Lafont S, Chiron M, Zins M, Lagarde E. Attitudes associated with behavioral predictors of serious road traffic crashes: result from the GAZEL cohort. Inj Prev 2007; 13:26-31.

39. Clarke DD, Ward P, Bartle C, Truman W. Young driver accidents in the UK: the influence of age, experience, and time of day. Accid Anal Prev 2006; 38:871-8.

40. Borowsky A, Shinar D, Oron-Gilad T. Age, skill, and hazard perception in driving. Accid Anal Prev 2010; 42:1240-9.

41. Argimón-Pallas JM, Jiménez-Villa J. Métodos de investigación clínica y epidemiológica. 4a Ed. Madrid: Elsevier; 2012.

Recibido el 02/Jun/2013

Versión final presentada el 12/Sep/2013

Aprobado el 17/Oct/2013 